\title{
Optical Fiber Review
}

Wissam Mahjoob Osman ${ }^{1 *}$, Amin Babiker Al Nabi' and Khalid Hammed Billal ${ }^{2}$

${ }^{1}$ Department of Communication, Faculty of Engineering, AL Neelain University, Khartoum, Sudan

${ }^{2}$ University of Science and Technology, Khartoum, Sudan

\begin{abstract}
With modern development, communication have become an important part of human life and cannot be dispensed with, the communication process involves information generation, transmission, reception and interpretation. Wide bandwidth for signal transmission with low delay is a key requirement in present day applications.

Fiber optics is now the transmission medium of data for long distance and it has high data rate transmission for Telecommunication networks. This paper gives an overview of fiber optic communication systems including the concept of wire communication, characteristic, architecture, optical fiber system (link budget design) and application of fiber optic.
\end{abstract}

Keywords: Fiber; Telecommunication networks; Electrical conductors; Local area networks

\section{Introduction}

Wired communication refers to based communication technology where data transmission over a wire for example twisted pair in low and high frequency and optical fiber in very high and ultra-high frequencies also telephone networks, television, internet Access and communication [1]. Also wave guide, used for high-Power applications and is considered as wired line [2]. Wireless communication includes the transferring of information over a range without cable or wire or any other shapes of electrical conductors. The distance traveled can be anyplace between a few meters (such as a TV remote control) and thousands of kilometers (such as, radio communication) [3]. Here we will focus in wired communication by using optical fiber. Optical fiber is a cylindrical dielectric made of Silica glass. There are a central core in which the light is guided, included in an outer Cladding of a little bit of lower refractive index [4] (Figure 1).

Communication indicate transmit of information from one point to another when it is needful. To transmit information such as image, speech, or data over a range, one generally uses the concept of carrier wave communication [5]. For gigabits and after gigabits for transference of data, fiber optics is the perfect choice. This type of communication is used to transfer video, voice and data over very long distances and local area networks (LAN) or computer networks. An optical fiber communication system uses light wave mechanism to transfer the data over a fiber by changing electronic signals into light [6] (Figure 2).

\section{Characteristic of optical fiber}

Fiber optics is a pioneer building block in the telecommunication infrastructure. Its high bandwidth efficiency and low attenuation features make it ideal for large transmission and beyond [7]. Optical-fiber systems have many advantages over mineral-based communication systems.

These advantages include attenuation, interference and bandwidth characteristics. Furthermore, the comparatively smaller cross section of fiber-optic cables let room for enormous growth of the capacity in existing tubes. Fiber-optic features can be categorized as linear and nonlinear. Nonlinear characteristics are affected by parameters such as power level, channel spacing, and bit rate [8].

\section{Interference}

Fiber optic cables doesn't affected by electromagnetic interference. It can also be immune of electrical noise in noisy environment [9].
From Computer Desktop Encyclopedia 1999 The Computer Language Co. Inc.

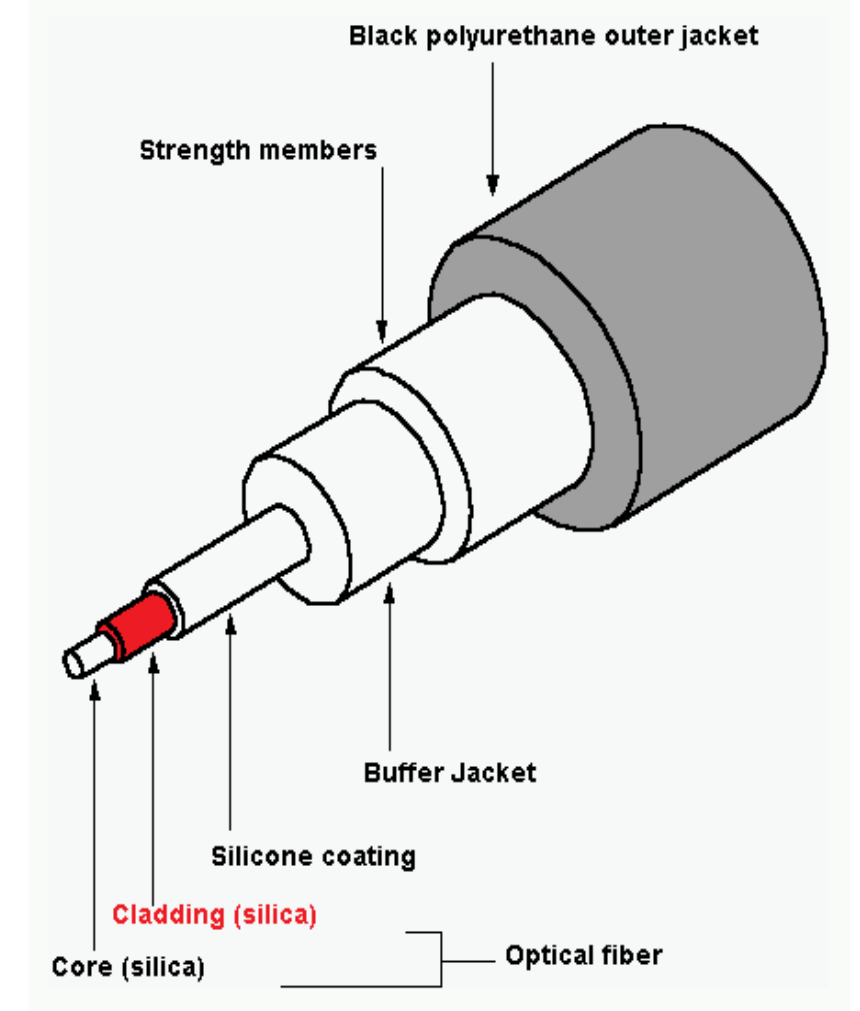

Figure 1: Optical fiber infrastructure.

*Corresponding author: Wissam Mahjoob Osman, Department of Communication, Faculty of Engineering, AL Neelain University, Khartoum, Sudan, Tel: 0915044840; E-mail: wisam.132@gmail.com

Received June 06, 2017; Accepted January 02, 2018; Published January 09 2018

Citation: Osman WM, AI Nabi AB, Billal KH (2018) Optical Fiber Review. J Electr Electron Syst 7: 249. doi: 10.4172/2332-0796.1000249

Copyright: (c) 2018 Osman WM, et al. This is an open-access article distributed under the terms of the Creative Commons Attribution License, which permits unrestricted use, distribution, and reproduction in any medium, provided the original author and source are credited. 


\section{Linear characteristic}

Linear characteristics include attenuation, chromatic dispersion (CD), polarization mode dispersion (PMD), and optical signal-tonoise ratio (OSNR).

\section{Attenuation}

Right works of an optical data link rely on modulated light arrived the receiver with enough power to be demodulated rightly. Attenuation is the decreasing in power of the light signal as it is transferred. Attenuation is occurs because passive media ingredients, for example cables, cable paste, and conductors. Although attenuation is huge scale down for optical fiber than for other media, it still happens in both multimode and single-mode send. An effective optical data link must have suffice light obtainable to defeat attenuation [10].

\section{Chromatic dispersion}

It is the outcome of the different wavelength or colors in a light beam arriving at their destination at partially different times. The result is a dispersion of the on-off light pulses that transmit digital information.

Special treatment must be considered to compensate for this dispersion so that the optical fiber reaches its maximum capacity [11].

Optical signal to noise ratio OSNR is very substantial parameters on physical layer of optical fiber system sending, pending fiber sending, amplified spontaneous emission (ASE) fuss is generated. It is a nonreversible influence that cannot be offset in optical domain immediately [12].

\section{Nonlinear characteristic}

Any increase in optical intensity in optical fiber leads to adjustment of refractive index and the wave propagation become a function of optical power. On the other hand, we find in linear fiber optics where the propagation constant is a function of fiber and the wavelength only the propagation constant becomes a function of optical power in addition to other parameters [13].

\section{Application of optical fiber}

Demand for optical fiber application has increased recently, because of increasing number of applications. Telecommunication applications are spread widely, start with universal networks and end with desktop computers. These involve the sending of data, sound, or video over distances between a meter to hundreds of kilometers, utilize one of a little bit of typical fiber designs in one of several cable designs [14].

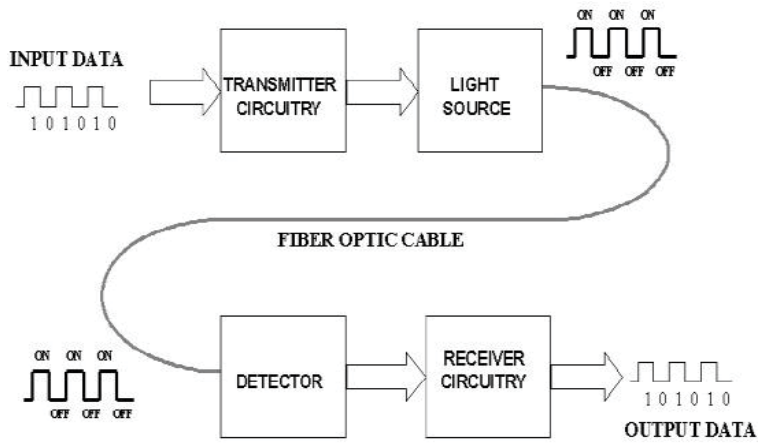

Figure 2: Data transferring by using optical fiber
CATV (cable television) services are provided by fiber optic network to an optical node, which transform and distributes the electrical signal to subscribers via a coaxial cable connection [7].

\section{Architectures}

Fiber-optic communication systems can be categorized into three broad categories-point-to-point links, distribution networks, and local-area networks.

\section{Point-to-point links}

Point-to-point links considered as the simplest form in optical fiber communication systems.

Their essential role is to transmit information in digital bit form from one place to another with high accuracy.

The length of link depends on required application. When the link length skip a certain value, reparations will be required rely on the operating wavelength to forbid the signal from coming too weak to be detected in effective way [15] (Figure 3).

\section{Distribution networks}

The optical devices and physical fiber that distribute signals to users in telecommunications network [16] (Figure 4).

\section{Local-area network}

A computer network covers a small area called LAN for example one site or building, such as a collage or a school [17] (Figure 5).

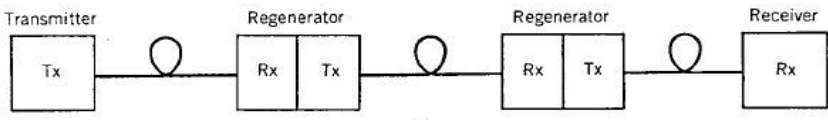

(a)

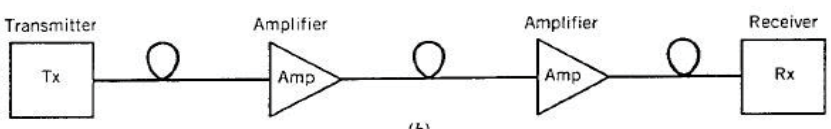

Figure 3: The point-to-point fiber links with cyclic loss reparations through (a) regenerators and (b) optical amplifiers. A regenerator contains a receiver then a transmitter.
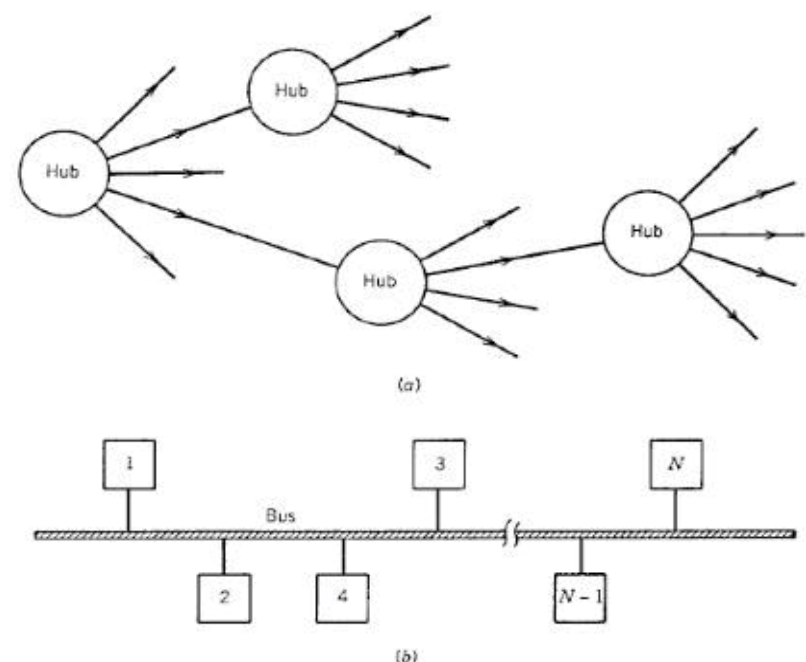

Figure 4: Distributed networks. 


\section{Wide area network}

Wide area network covers a geographically large area and called (WAN). A wan consisting of two or more local area networks (LAN) or metro area networks (MAN) and is simply a scattered communication network for transmission of data, image, video, voice, and so on [18] (Figure 6).

\section{Optical Fiber System (Link Budget Design)}

The main goal of power budget is to provide enough power to reach the receiver to maintain reliable performance during the entire system lifetime. The receiver sensitivity is a minimum average power required by the receiver. The average launch power is mostly specified for each transmitter with optical powers expressed in dB [19-24].

Fiber-optic connections must have enough power for correct operation, and for doing that, power budget must be calculated, which is the maximum amount of power it can be send.

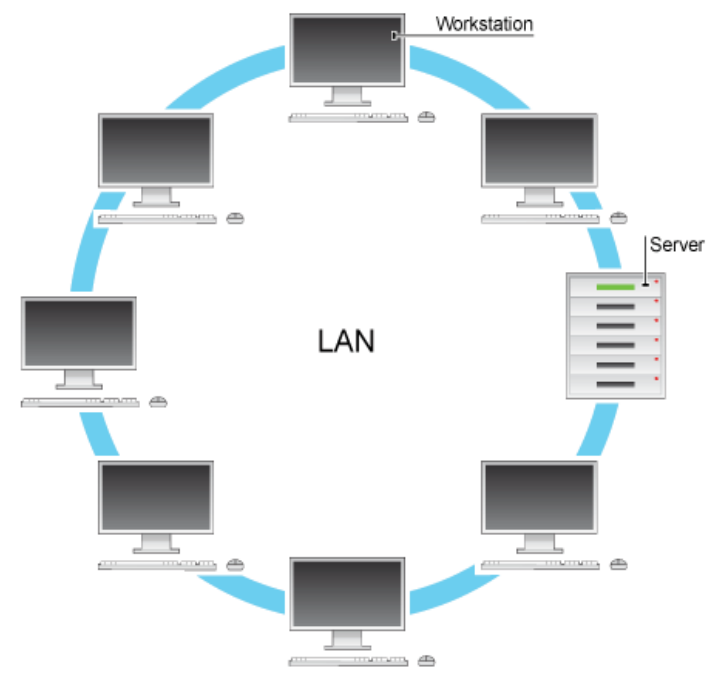

Figure 5: LAN (Local Area Network).

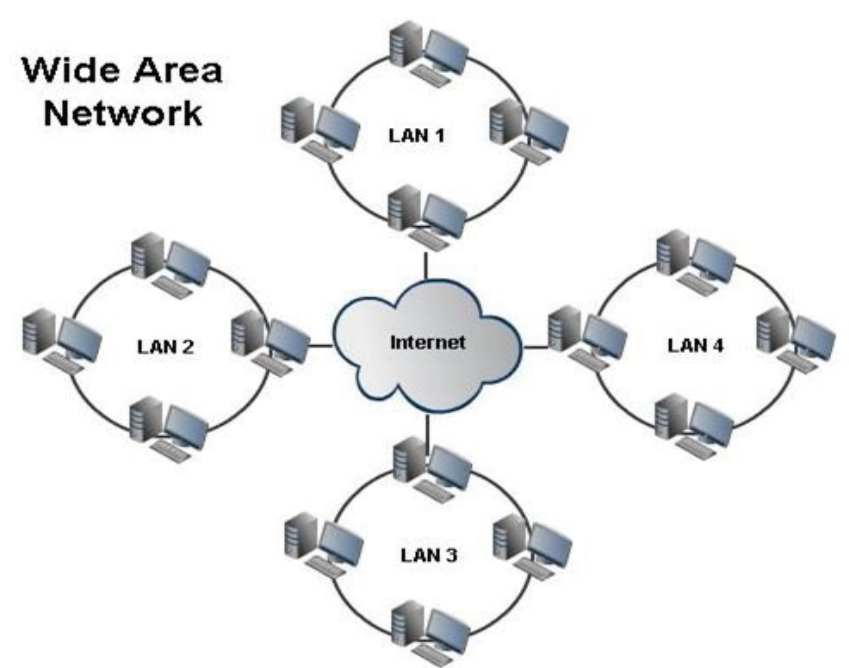

Figure 6: WAN (Wide Area Network).
The bad case is used to find out the power budget analysis to supply an error margin, although all the parts of an actual system do not function at the worst-case levels.

To find out the bad-case estimation of power budget (PB), you assume less transmitter power (PT) and minimum receiver sensitivity (PR):

$\mathrm{PB}=\mathrm{PT}-\mathrm{PR}$

The next assumption power budget equation uses values measured in decibels $(\mathrm{dB})$ and decibels refer to one mill watt $(\mathrm{dBm})[20]$ :

$$
\begin{aligned}
& \mathrm{PB}=\mathrm{PT}-\mathrm{PR} \\
& \mathrm{PB}=-15 \mathrm{dBm}-(-28 \mathrm{dBm})
\end{aligned}
$$

$\mathrm{PB}=13 \mathrm{~dB}$

\section{Previous Studies}

Jadhav and Shitole published a scientific paper, entitled "Fiber optic communication and application" they took a detailed look at the technological advantages of a fiber optic telecommunication Network and its applications [21].

The same work was also done by Xue-Zhao [22] entitled "Method for dispersion on optical fiber communication with long distances", he focused on paper on dispersion on optical fiber communication. On the other hand Sabah Al-Bazzaz [23] presented simulation methods on a single mode optical fiber link system, using $\mathrm{VC}++$ in his published scientific paper entitled "Simulation of Single Mode of Fiber Optics and Optical Communication Components Using VC++". While Sharma et al. published scientific paper entitled "Fiber Optic Communications: An Over view, Deals with communication using optical fibers" [24].

\section{Conclusions}

This paper presented a detailed look at the communication concept for wire, in particular the characteristic and application of fiber optic also we focus at the architecture and optical fiber system (link budget design).

There are a huge amount of development can be made by making more research's and work on optical fiber.

We need it for a faster and more reliable infrastructure which would be the prime demand of the ever growing population of tomorrow.

\section{References}

1. Ali-Mohamed AY, Jaffar AH (2000) Estimation of atmospheric inorganic watersoluble aerosols in the western region of Bahrain by ion chromatography. Chemosphere 2: 85-94

2. https://en.wikipedia.org/wiki/Wired_communication

3. Rose Mary (2010) Wireless communication and types. Engineers Garage.

4. Saleh BEA, Teich MC (1991) Fiber optic. Fundamentals of Photonics.

5. Hull D, Souders J (2008) Principles of fiber optic communication. Waco, Texas: CORD Communications.

6. Agarwal T (2010) What are the Basic Elements of a Fiber Optic Communication System? The Budding electronic Engineer's Knowledge Space.

7. Abbas TZ (2011-2012) Fiber optics.

8. Vivek Alwayn (2004) Fiber-optic technologies. CISCO.

9. http://www.services.eng.uts.edu.au/ akadi/ite/major_assignments/barber/ advdisad.htm

10. https://www.juniper.net/documentation/en_US/release-independent/ junos/topics/concept/fiber-optic-cable-signal-loss-attenuation-dispersionunderstanding.html 
11. http://www.m2optics.com/blog/bid/61431/Chromatic-Dispersion-in-OpticalFibers

12. Yu Y, Zhang B, Yu C (2014) Optical signal to noise ratio monitoring using single channel sampling technique. OSA 22: 6874-6880.

13. Basics of non-linear fiber optics.

14. https://www.slideshare.net/Rimmi07/application-of-fibre-optics-incommunication

15. Architectures of fiber-optic communication systems. Communication through optical fibres.

16. https://en.wikipedia.org/wiki/Electric_power_distribution

17. Networks and communication.

18. https://www.mbaskool.com/business-concepts/it-and-systems/13445-wan. $\mathrm{html}$
19. System design-power budget.

20. https://www.juniper.net/documentation/en_US/release-independent/junos/ topics/task/installation/fiber-optic-cable-budget-margin-calculating.html

21. Jadhav RA, Shitole DS (2013) Fiber optic communication and application. International Journal of Innovative Research in Engineering and Science.

22. Xue-Zhao Z (2014) Method for dispersion on optical fiber communication with long distances. International Journal of Future Generation Communication and Networking 7: 1-12.

23. Al-Bazzaz SHS (2008) Simulation of single mode fiber optics and optical communication components using VC++. International Journal of Computer Science and Network Security 8: 300-308.

24. Sharma P, Arora RK, Pardeshi S, Singh M (2013) Fiber optic communications: an over view. International Journal of Emerging Technology and Advanced Engineering 3: 474-479. 